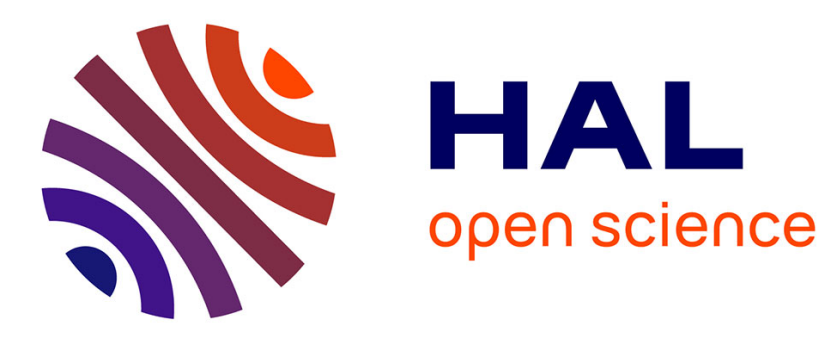

\title{
Les faisceaux du VIVITRON
}

A. Nadji, F. Haas, G. Heng, J. Hoffmann, Ch. Muller, R. Rebmeister

\section{To cite this version:}

A. Nadji, F. Haas, G. Heng, J. Hoffmann, Ch. Muller, et al.. Les faisceaux du VIVITRON. Revue de Physique Appliquée, 1988, 23 (9), pp.1445-1451. 10.1051/rphysap:019880023090144500 . jpa00245969

\section{HAL Id: jpa-00245969 https://hal.science/jpa-00245969}

Submitted on 1 Jan 1988

HAL is a multi-disciplinary open access archive for the deposit and dissemination of scientific research documents, whether they are published or not. The documents may come from teaching and research institutions in France or abroad, or from public or private research centers.
L'archive ouverte pluridisciplinaire HAL, est destinée au dépôt et à la diffusion de documents scientifiques de niveau recherche, publiés ou non, émanant des établissements d'enseignement et de recherche français ou étrangers, des laboratoires publics ou privés. 
Classification

Physics Abstracts

$29.15 \mathrm{~B}-29.25 \mathrm{C}-29.25 \mathrm{~F}$

\title{
Les faisceaux du VIVITRON
}

\author{
A. Nadji, F. Haas, G. Heng, J. Hoffmann, Ch. Muller et R. Rebmeister \\ Centre de Recherches Nucléaires (I N2 P3), 67037 Strasbourg Cedex, France
}

(Reçu le 15 octobre 1987, accepté le 17 mai 1988)

\begin{abstract}
Résumé. - Dans le VIVITRON, il est prévu d'accélérer une cinquantaine de faisceaux de nature différente et d'énergie variable pouvant atteindre $20 \mathrm{MeV} / \mathrm{n}$ pour les ions lourds « légers » et $5 \mathrm{MeV} / \mathrm{n}$ pour les ions les plus lourds $(A \sim 200)$. L'obtention de ces faisceaux avec les excellentes qualités propres aux machines électrostatiques nécessite cependant la mise en cuvre d'une sélection fine en masse à l'injecteur, d'une sélection en charge après épluchage (gaz ou feuille) au niveau de l'électrode centrale ainsi qu'une sélection en charge et en masse dans un aimant d'analyse placé après la machine et ayant des capacités de déviation adaptées aux faisceaux délivrés $(K=500)$.

Abstract. - In the VIVITRON, it is planned to accelerate about fifty beams of different nature and variable energy between $20 \mathrm{MeV} / \mathrm{n}$ for «light » heavy ions and $5 \mathrm{MeV} / \mathrm{n}$ for the heaviest $(A \sim 200)$. To obtain these beams with the excellent qualities typical of electrostatic accelerators requires however a fine mass resolution at the injector, a charge selection after stripping (gaz or foil) in the terminal as well as charge and mass selection in the $90^{\circ}$ energy analysing magnet, placed after the accelerator. The magnet will have bending capacities adapted to the delivered beams $(K=500)$.
\end{abstract}

\section{Introduction.}

L'accélérateur électrostatique VIVITRON [1] est apte à fournir des tensions élevées et des faisceaux de qualité jamais atteinte dans une grande partie de la plage d'énergies comprises entre 40 et $800 \mathrm{MeV}$. Il constitue certainement, dans le domaine d'énergie pour lequel il est construit, la machine la plus performante.

Les caractéristiques principales des faisceaux produits sont une variété importante de projectiles, une très bonne définition géométrique, une excellente résolution en énergie et la possibilité de changer rapidement l'énergie des projectiles.

Pour exploiter pleinement ces propriétés, une étude détaillée du transport du faisceau entre la source d'ions et la cible est nécessaire. Le travail que nous exposons ici représente les points les plus importants de l'état actuel des calculs.

Dans une première partie, nous allons nous intéresser à l'analyse en masse à l'injecteur ainsi qu'à l'énergie de préaccélération nécessaire à une focalisation adéquate à l'entrée du premier tube accélérateur et à une bonne transmission du faisceau à travers la section basse énergie de la machine. La sélection en état de charge à l'électrode terminale sera décrite dans la deuxième partie de ce travail. Le rôle du sélecteur consiste non seulement à écarter les états de charge indésirables mais aussi à améliorer l'adaptation et donc la transmission du faisceau à travers la section haute énergie. La troisième partie portera sur une description succincte de l'analyse en énergie du faisceau après accélération et de son transfert vers les aires expérimentales.

\section{Le faisceau avant le VIVITRON : l'injecteur.}

L'injecteur du Tandem MP actuel comprend 2 voies analysées par un dipôle de $\pm 35^{\circ}$. Le pouvoir séparateur pratique de cet équipement est modeste $\frac{\Delta m}{m} \sim \frac{1}{20}$ pour un diamètre objet et image égal à $4 \mathrm{~mm}$. Rajouter une voie centrale avec sa propre analyse, constitue une solution performante et économique. Un calcul au premier ordre, effectué avec le programme TRANSPORT [2], a donné un pouvoir séparateur d'environ $\frac{1}{200}$ [3]. L'énergie d'injection retenue est de $250 \mathrm{keV}$ qui se décompose de la façon suivante : une énergie de $20 \mathrm{keV}$ provenant de la tension d'extraction de la source, une énergie de $150 \mathrm{keV}$ fournie par l'actuel tube accélérateur alimenté par la plate-forme haute tension existante et enfin une énergie de $80 \mathrm{keV}$ délivrée par un tube 
supplémentaire installé en amiont du dipôle d'analyse.

A cette énergie, les oscillations verticales prenant naissance dans la première section du tube $\mathrm{n}^{\circ} 1$ à champ incliné du VIVITRON sont maintenues à une valeur acceptable $( \pm 3 \mathrm{~mm})$ grâce à une injection légèrement inclinée ( $15 \mathrm{mrad})$ dans le plan vertical. Le schéma de principe de l'injecteur est reporté sur la figure 1. Le faisceau émis par la source est refocalisé par une lentille électrostatique en un point représentant le point objet de l'injecteur et situé $34 \mathrm{~cm}$ en aval de la source. L'enveloppe du faisceau dans la nouvelle section est montrée sur la figure 2 .

Cette réalisation permet de conserver au faisceau sa symétrie de révolution, de diminuer l'émittance du faisceau d'un facteur $\sqrt{5}$, de réduire la charge d'espace et enfin de bénéficier d'une section efficace de neutralisation des ions négatifs plus faible. En outre, l'utilisation d'une source à «sputtering » ayant une bonne brillance (rapport intensité/émittance) rend ce système très performant.

\section{Le faisceau à l'intérieur de la machine.}

3.1 SECTION BASSE ÉNERGIE. - Les faisceaux d'ions négatifs émis par l'injecteur, sont focalisés sur un diaphragme de position fixe situé à $2,5 \mathrm{~m}$ de l'entrée du premier tube de l'accélérateur. Ce diaphragme matérialise le point objet de l'ensemble optique composé d'une lentille grille ajustable disposée à l'entrée du tube $n^{\circ} 1$ et de la lentille naturelle existant $50,8 \mathrm{~cm}$ en aval, à la jonction des électrodes fonctionnant à demi-champ et de celles fonctionnant à champ nominal. A cet endroit, est également placé le diaphragme d'entrée $(\varnothing=28 \mathrm{~mm})$, le diaphragme de sortie étant constitué par le canal d'épluchage $(\varnothing=8 \mathrm{~mm})$.

Le tube $\mathrm{n}^{\circ} 1$. - La section accélératrice d'entrée (tube $n^{\circ} 1$ ) est traitée spécialement. En effet, cette section a été calculée de façon à éliminer la partie à champ droit correspondant à la limitation observée sur le Tandem MP. Une étude spéciale a conduit J. D. Larson [4] à subdiviser ce premier tube en deux parties. La première, longue de $50,8 \mathrm{~cm}$, comporte un dosage adéquat d'électrodes inclinées à $\pm 7^{\circ}, 0^{\circ}$, et $\pm 14^{\circ}$, le champ inter-électrode est moitié du champ nominal. La deuxième partie fonctionnant au gradient nominal est longue de $106,7 \mathrm{~cm}$ et l'inclinaison des électrodes est constante $\left(14^{\circ}\right)$. Cette géométrie de tube à champ incliné permet de minimiser l'excursion du faisceau dans les limites de

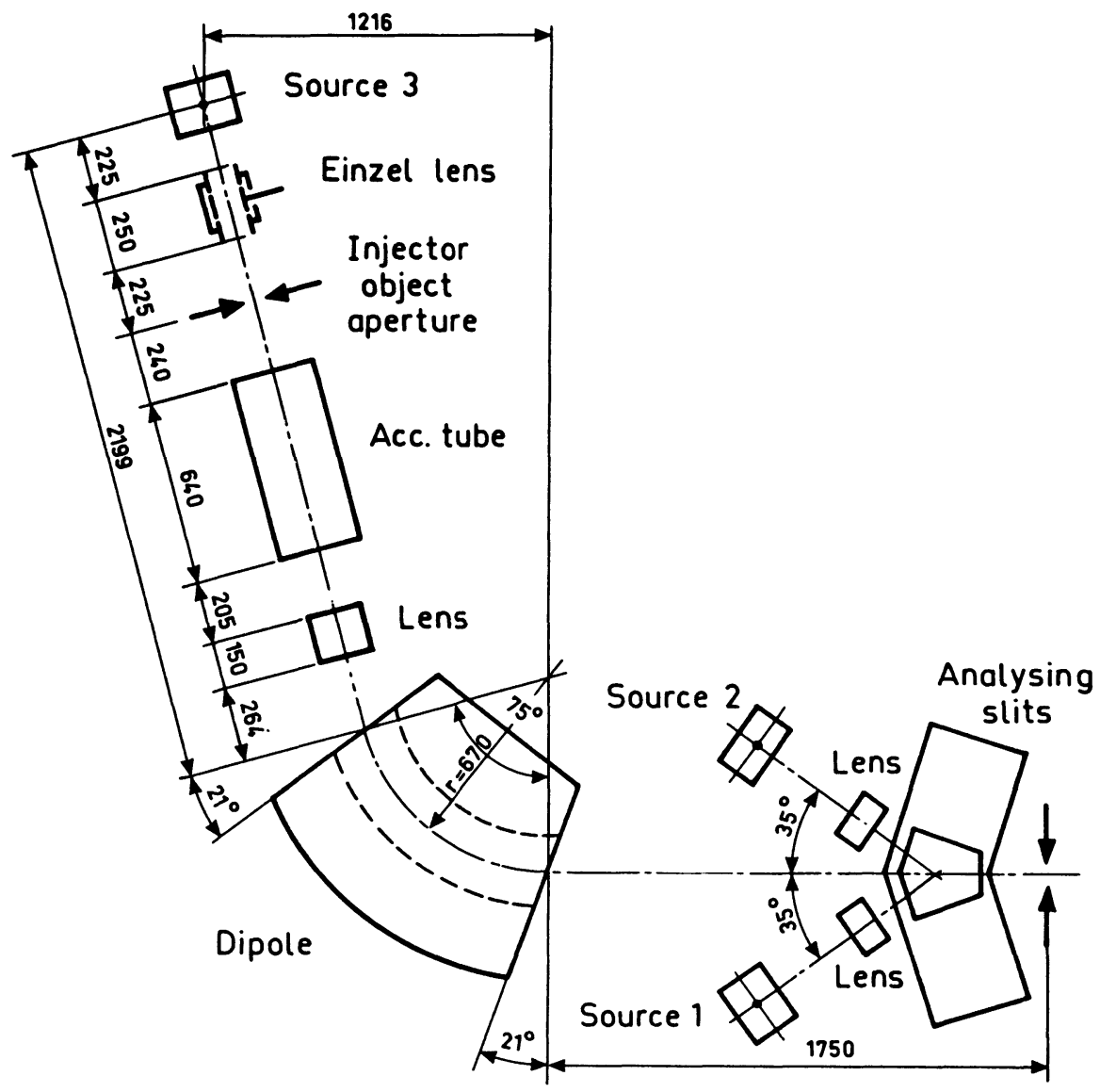

Fig. 1. - Représentation schématique de l'injecteur du VIVITRON.

[Schematic representation of the VIVITRON injector.] 
VERTICAL -

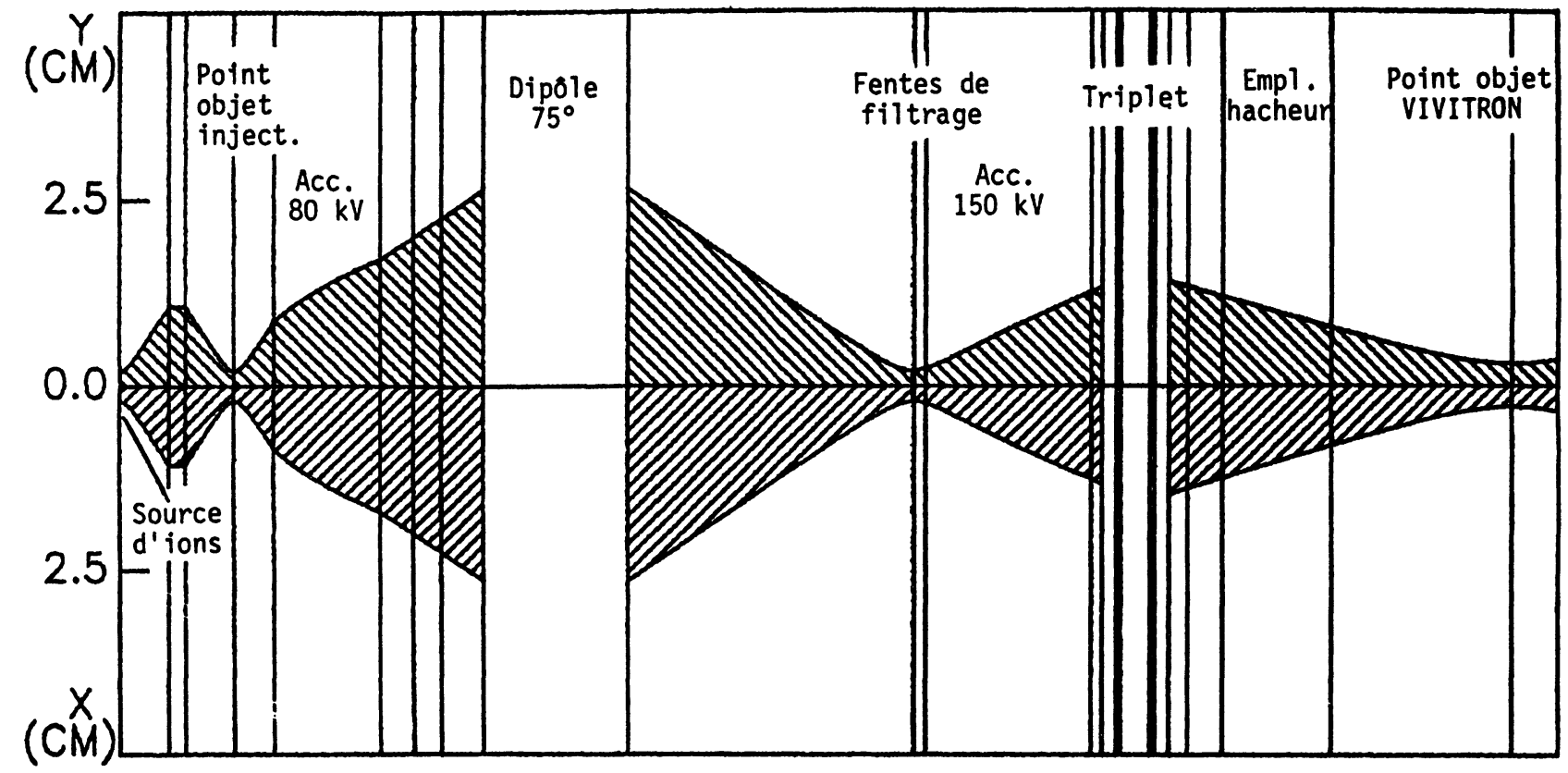

HORIZONTAL

Fig. 2. - Enveloppes du faisceau entre la source d'ions et le point objet du VIVITRON dans la troisième voie de l'injecteur.

[Beam envelopes between the ion source and the object point of the VIVITRON along the central channel of the injector.]

$\pm 3 \mathrm{~mm}$. A la sortie du tube $\mathrm{n}^{\circ} 1$, le faisceau se retrouve sur l'axe du tube.

Transmission dans la section basse énergie. - On a admis dans nos calculs, une émittance normalisée du faisceau correspondant à $90 \%$ de son intensité : $E_{\mathrm{n}}=10 \pi . \mathrm{mm} \cdot \mathrm{mrad} \sqrt{\mathrm{MeV}}$. A l'énergie d'injection retenue de $250 \mathrm{keV}$, cette émittance est de $20 \pi . \mathrm{mm}$. mrad. Les acceptances horizontale $A_{\mathrm{H}}$ et verticale $A_{\mathrm{V}}$ de la section, calculées à partir des formules données dans la référence [5] sont toujours inférieures à l'émittance du faisceau injecté. En effet :

pour $U_{\mathrm{M}}=20 \mathrm{MV}$, on a

$$
A_{\mathrm{H}}=12,2 \pi \mathrm{mm} \mathrm{mrad}, A_{\mathrm{V}}=9,6 \pi \mathrm{mm} \mathrm{mrad}
$$

et pour $U_{\mathrm{M}}=35 \mathrm{MV}$, on a

$A_{\mathrm{H}}=15,7 \pi \mathrm{mm} \mathrm{mrad}, A_{\mathrm{V}}=12,3 \pi \mathrm{mm} \mathrm{mrad}$.

On constate donc que le faisceau ne peut pas être transmis intégralement. Pour améliorer cette situation, plusieurs solutions peuvent être envisagées : utiliser des sources plus brillantes, agrandir les ouvertures dans les électrodes du tube $n^{\circ} 1$ ou encore augmenter le diamètre du canal d'épluchage.

3.2 EleCtrode TERMinAle ET SÉleCtEuR DE CHARGE. - Après avoir traversé l'éplucheur de REVUE DE PHYSIQUE APPLIQUÉE. - T. 23, N 9, SEPTEMBRE 1988 l'électrode terminale, le faisceau comporte un grand éventail d'états de charge dont un seul sera utilisé. Une fraction des autres faisceaux, qui d'ailleurs peut être plus intense que le faisceau sélectionné, va heurter des électrodes du tube et créer ainsi des électrons secondaires dont la production excessive peut être néfaste au bon fonctionnement de la machine. Dans un accélérateur comme le VIVITRON, avec une tension à l'électrode terminale de $35 \mathrm{MV}$ et utilisant dans la plupart des cas des faisceaux d'ions lourds, un sélecteur d'états de charge capable d'arrêter toutes les charges non désirées et de transmettre sans perte les charges sélectionnées, sera un équipement d'une grande utilité.

Ce sélecteur d'états de charge doit avoir une sélectivité élevée et doit préserver ou même améliorer l'optique du faisceau sélectionné.

La solution la plus compatible avec le VIVITRON est un triplet déplacé de quadrupôles électrostatiques qui combine la sélection et l'adaptation [6]. Cette lentille désaxée va créer une dispersion angulaire des faisceaux selon les différents états de charge, un seul revenant sur l'axe de la machine et étant refocalisé sur un diaphragme placé devant le second éplucheur. La figure 3 illustre le principe de fonctionnement de ce triplet.

D'autres calculs sont en cours pour trouver la 


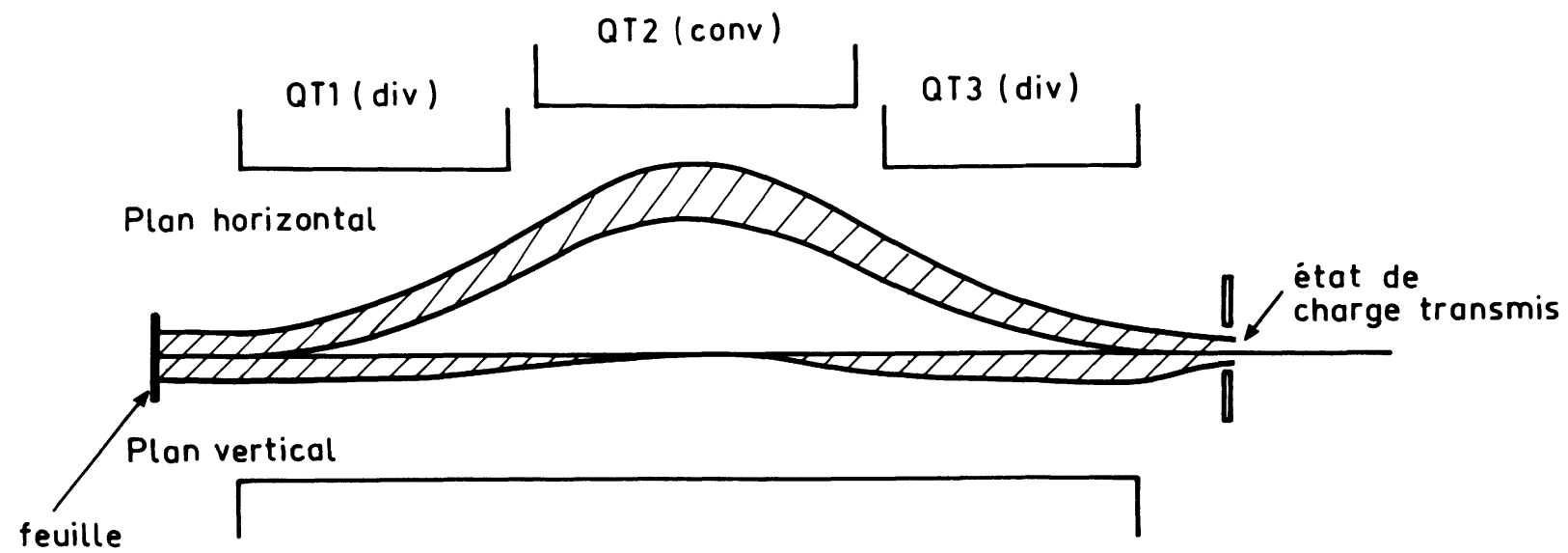

Fig. 3. - Principe de fonctionnement d'un sélecteur de charge : QT1 et QT3 : deux quadrupôles divergents dans le plan horizontal (plan de dispersion). QT2 : quadrupôle convergent dans ce même plan.

[Principles of operation of the charge state selector.]

meilleure disposition possible du triplet, c'est-à-dire celle qui donne la meilleure dispersion des faisceaux non désirés.

3.3 Section haute Énergie. - A la sortie de l'éplucheur central, les qualités du faisceau d'ions sont dégradées. Il a subi une dispersion angulaire qui augmente son émittance transversale, ainsi qu'une perte et une dispersion en énergie qui augmentent son émittance longitudinale. Dans cette section, le faisceau est partout divergent. Les diaphragmes limitant l'acceptance de cette section sont le canal d'épluchage déjà cité et un diaphragme circulaire $(\varnothing=28 \mathrm{~mm})$ situé à la sortie du dernier tube. L'amplitude des oscillations verticales dues aux électrodes inclinées est toujours inférieure à $1 \mathrm{~mm}$ (situation meilleure que pour la section basse énergie).

Transmission à travers la section haute énergie. En ne tenant pas compte des pertes de la section basse énergie et en considérant deux tensions extrêmes de la machine, les émittances des faisceaux et l'acceptance de la section haute énergie se comparent de la façon indiquée dans le tableau I.

Si l'on ne tient pas compte de l'effet du vide dans le tube, le bilan global de la transmission du VIVITRON est résumé dans le tableau II, [7].

Le calcul de transmission des faisceaux à travers le VIVITRON (VIV) donne des valeurs relativement

Tableau I.

\begin{tabular}{ccccc}
\hline$U_{\mathrm{M}}(\mathrm{MV})$ & $\begin{array}{c}\text { Emittance à l'entrée } \\
\text { de la section HE } \\
(\mathrm{mm} . \mathrm{mrad})\end{array}$ & $\begin{array}{c}\text { Etat de charge } \\
\text { sélectionné }\end{array}$ & $\begin{array}{c}\text { Emittance à la sortie } \\
\text { de la section HE } \\
(\mathrm{mm} . \mathrm{mrad})\end{array}$ & $\begin{array}{c}\text { Acceptance } \\
\text { de la section HE } \\
(\mathrm{mm} . \mathrm{mrad})\end{array}$ \\
\hline 20 & $2,2 \pi$ & $13^{+}$ & $8 \pi$ & $5,7 \pi$ \\
35 & $1,7 \pi$ & $6^{+}$ & $1,7 \pi$ & $4,4 \pi$ \\
\hline
\end{tabular}

Tableau II.

\begin{tabular}{ccccc}
\hline & $\begin{array}{c}\text { Type de distribution } \\
\text { de l'intensité/émittance }\end{array}$ & \multicolumn{2}{c}{ Transmission } \\
\cline { 3 - 5 }$U_{\mathrm{M}}=20 \mathrm{MV}, A=130, Q=13^{+}$ & Linéaire & $\mathrm{BE}$ & HE & VIVITRON \\
\hline \multirow{2}{*}{$U_{\mathrm{M}}=35 \mathrm{MV}, A=12, Q=6^{+}$} & Gaussienne & 0,32 & 0,50 & 0,16 \\
& Linéaire & 0,52 & 0,65 & 0,34 \\
\hline
\end{tabular}


faibles. En effet, l'accélérateur VIV étant plus long que le Tandem MP (51 m/25 m), son acceptance sera plus petite. Chaque section d'accélération du VIV est approximativement 2,3 fois plus longue que son homologue du MP. Sans accélération, cette augmentation de longueur réduit l'acceptance (pour une même ouverture d'électrode) d'un facteur $\frac{1}{2,3}=$ 0,43 par rapport à celle du MP. Une accélération dans un champ de même gradient limite cette réduction à un facteur $\frac{1}{\sqrt{2,3}}=0,65$. L'effet engendré par cette acceptance réduite sur la transmission du faisceau dépendra des caractéristiques du faisceau injecté dans l'accélérateur. Il est très important (comme on l'a déjà mentionné précédemment) que pour le VIV soient développées des sources ayant une grande brillance.

Des enveloppes du faisceau du point objet du VIV au point objet de l'aimant d'analyse sont reportées sur la figure 4.

\section{Le faisceau après la machine et son transfert vers les cibles.}

A la sortie de l'accélérateur, le faisceau est divergent. Il est alors refocalisé par un doublet quadrupolaire magnétique pour passer dans les lèvres « objet » de l'aimant d'analyse de $90^{\circ}$ et de $K=500$.

La figure 5 schématise le système de transport du VIVITRON.

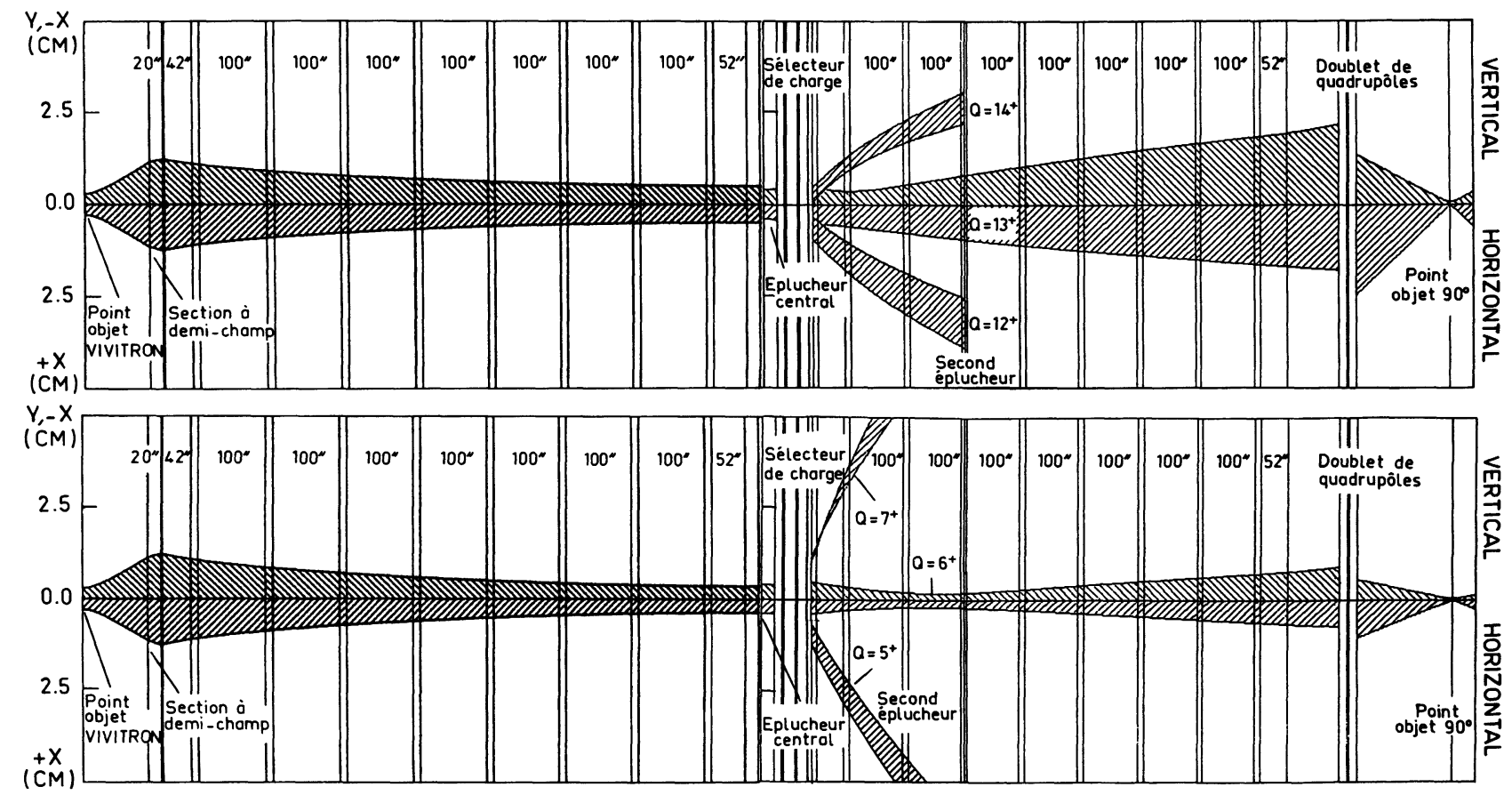

Fig. 4. - Enveloppes du faisceau du point objet du VIVITRON au point objet de l'aimant d'analyse. Figure du haut : tension de la machine $=20 \mathrm{MV}, A=130, Q=12^{+}, 13^{+}, 14^{+}$. Figure du bas : tension de la machine $=35 \mathrm{MV}$, $A=14, Q=5^{+}, 6^{+}, 7^{+}$.

[Beam envelopes between the object points of the VIVITRON and of the analysing magnet.]

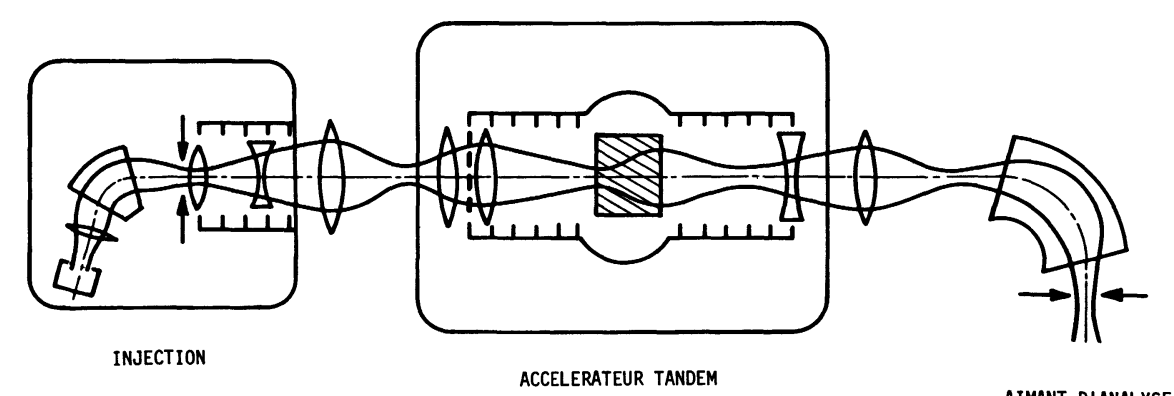

AIMANT D'ANALYSE

Fig. 5. - Représentation schématique du système de transport du faisceau à travers l'accélérateur VIVITRON.

[Schematic representation of the beam transport system through the VIVITRON accelerator.] 


\subsection{ANALYSE DES CARACTÉRISTIQUES DU FAIS- CEAU TRANSFÉRÉ VERS LES CIBLES.}

Energie. - La figure 6 montre, en fonction de la 'masse des ions accélérés, l'énergie par nucléon obtenue à $U=35 \mathrm{MV}$ selon le type d'éplucheur utilisé. En résumé :

- des faisceaux de $\mathrm{H}$, He et de Li peuvent être produits à 70,105 et $140 \mathrm{MeV}$ respectivement ;

- des faisceaux d'ions lourds « légers » $(\mathrm{C}$ à $\mathrm{Ca})$ avec un éplucheur à gaz auront de 10 à $16 \mathrm{MeV} / \mathrm{n}$ et avec un éplucheur à feuille, de 15 à $20 \mathrm{MeV} / \mathrm{n}$;

- pour des ions mi-lourds (Ca à I), il faut utiliser l'épluchage à feuille pour avoir de 6 à $10 \mathrm{MeV} / \mathrm{n}$, avec un double épluchage feuille-feuille on atteint 10 à $15 \mathrm{MeV} / \mathrm{n}$;

- les ions très lourds auront des énergies de l'ordre de $5 \mathrm{MeV} / \mathrm{n}$ pour un épluchage feuillefeuille.

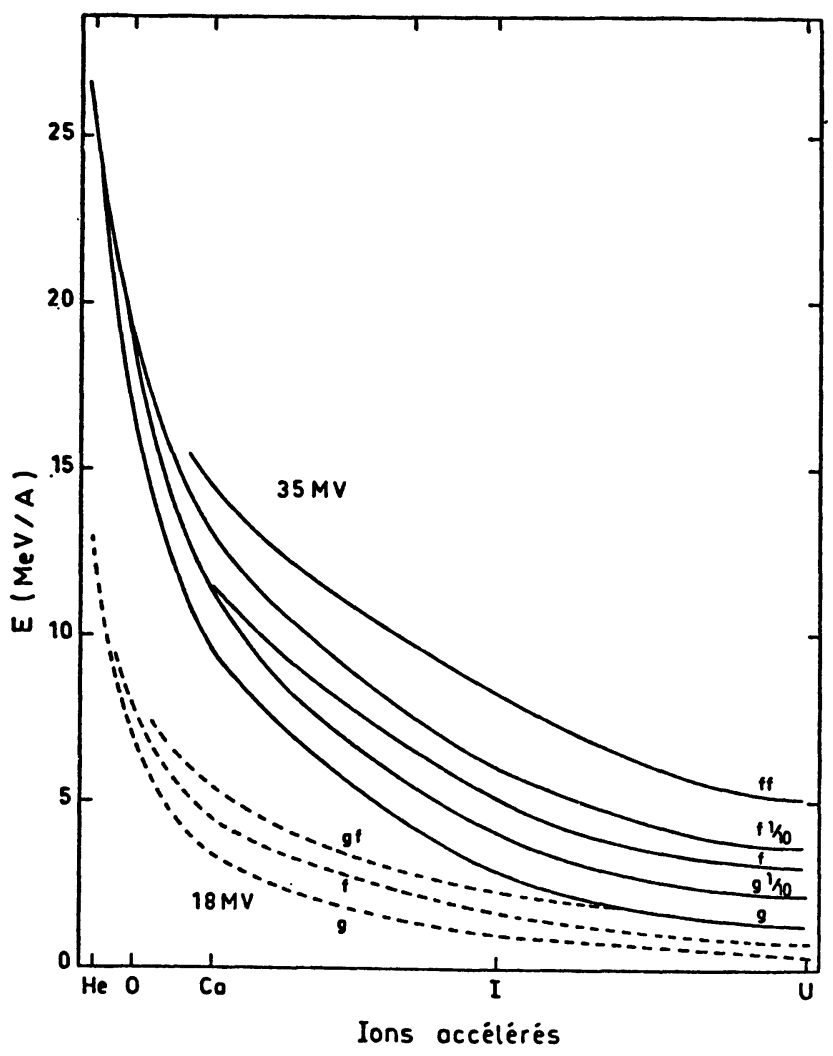

Fig. 6. - Performances en énergie du VIVITRON pour une tension de $35 \mathrm{MV}$ correspondant à un fonctionnement sous différentes conditions d'épluchage.

[Beam energies of the VIVITRON at a terminal voltage of $35 \mathrm{MV}$ under different stripping conditions.]
Intensité. - L'intensité du faisceau accéléré dépend de plusieurs paramètres : l'intensité initiale des ions négatifs fournis par la source, le rendement de l'épluchage, l'augmentation d'émittance due au phénomène de dispersion angulaire dans l'éplucheur et la transmission à travers l'accélérateur. La figure 7 donne quelques indications sur l'évolution de l'intensité des ions accélérés pour différentes conditions d'épluchage.

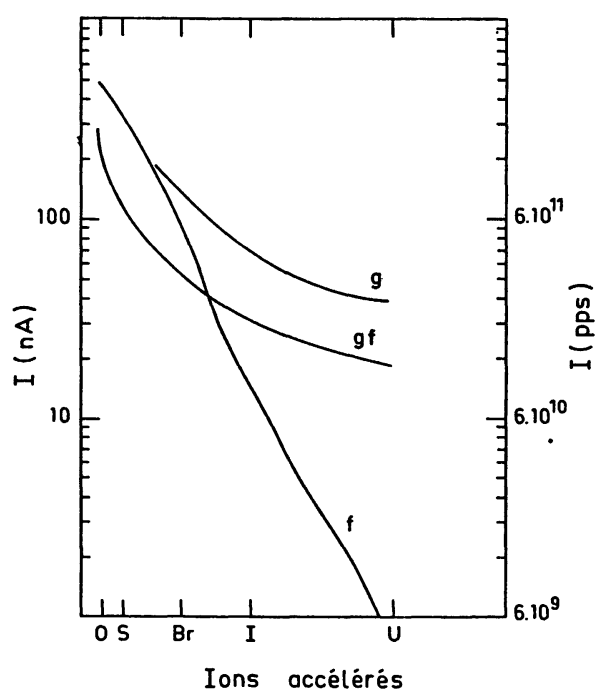

Fig. 7. - Intensités des faisceaux produits à l'accélérateur VIVITRON pour différentes conditions d'épluchage.

[Beam intensities of the VIVITRON under different stripping conditions.]

\section{Conclusion.}

Une étude au premier ordre de l'optique du faisceau du VIVITRON a été réalisée pour montrer la faisabilité et la flexibilité des différentes solutions proposées.

Les résultats trouvés sont valables pour une émittance normalisée de $10 \pi \mathrm{mm} . \mathrm{mrad} \sqrt{\mathrm{MeV}}$ et ne tiennent compte ni de l'effet du vide, ni de l'effet de la diffusion multiple due à l'interaction du faisceau avec l'éplucheur.

Une étude systématique basée sur des mesures d'émittances des différentes sources existantes au C.R.N. est en cours pour améliorer les qualités du faisceau à l'injection et par conséquent améliorer sa transmission à travers l'accélérateur. 


\section{Bibliographie}

[1] Le VIVITRON, Rapp. CRN-VIV-12 (1983).

[2] Brown, K. L., Carey, D. C., Iselin, Ch. and ROTHACKER, F., TRANSPORT : a computer program for designing charged particle beam transport systems, CERN 80-84. Rep.

[3] haas, F., Heng, G., Hoffmann, J., Muller, Ch. and REBMEISTER, R., The injector for the $35 \mathrm{MV}$ Tandem Accelerator VIVITRON of Strasbourg, Rapp. CRN-VIV-35 (1987).
[4] Larson, J. D., Nucl. Instrum. Methods A 244 (1986) 192.

[5] LARSON, J. D. and JoNeS, C. M., Nucl. Instrum. Methods 140 (1977) 489.

[6] NaDJI, A., Contribution à l'étude d'un sélecteur de charge pour le VIVITRON, Rapp. CRN-VIV25 (1986).

[7] REBMEISTER, R., Le faisceau à l'intérieur du VIVITRON, Rapp. CRN-VIV-34 (1986). 